\title{
Erratum: Cramér-Rao bound for time-continuous measurements in linear Gaussian quantum systems [Phys. Rev. A 95, 012116 (2017)]
}

\author{
Marco G. Genoni \\ (Received 3 May 2017; published 23 May 2017)
}

DOI: 10.1103/PhysRevA.95.059908

There is a term missing in one of the formulas in Eqs. (15). The derivative of the first moments vector with respect to the parameter $\theta$ should read

$$
\begin{aligned}
d\left(\partial_{\theta} \mathbf{R}_{t}\right)= & \left(\partial_{\theta} A\right) \mathbf{R}_{t} d t+A\left(\partial_{\theta} \mathbf{R}_{t}\right) d t+\left(\partial_{\theta} \mathbf{u}\right) d t \\
& +\frac{\left(\partial_{\theta} \boldsymbol{\sigma}_{t}\right) B}{\sqrt{2}} \mathbf{d w}+\left(\frac{\boldsymbol{\sigma}_{t} B+N}{\sqrt{2}}\right)\left(\partial_{\theta} \mathbf{d w}\right) .
\end{aligned}
$$

The vector of Wiener increments as reported in the Appendix B depends on the difference between the measured outcome $\mathbf{x}_{m}$ and its mean value and can be written as

$$
\mathbf{d w}=\left(\frac{\boldsymbol{\sigma}_{b}+\boldsymbol{\sigma}_{m}}{2}\right)^{-1 / 2}\left(\mathbf{x}_{m}-\overline{\mathbf{x}}_{m}\right) d W .
$$

As the mean value $\overline{\mathbf{x}}_{m}$ depends on the parameter $\theta$, its derivative is not zero, and one obtains

$$
\begin{aligned}
\partial_{\theta} \mathbf{d w} & =-\left(\frac{\boldsymbol{\sigma}_{b}+\boldsymbol{\sigma}_{m}}{2}\right)^{-1 / 2}\left(\partial_{\theta} \overline{\mathbf{x}}_{m}\right) d W \\
& =-\left(\frac{\boldsymbol{\sigma}_{b}+\boldsymbol{\sigma}_{m}}{2}\right)^{-1 / 2} \Omega C^{\top}\left(\partial_{\theta} \mathbf{R}_{t}\right) d t \\
& =\sqrt{2} B^{\top}\left(\partial_{\theta} \mathbf{R}_{t}\right) d t .
\end{aligned}
$$

The Fisher information (FI) can then be calculated as explained in the paper, and the effectiveness of the method presented is not affected by this error. However considering the extra term in Eq. (1) affects the numerical results obtained for the two examples described in the paper.

With regard to the estimation of the squeezing parameter for a quantum parametric amplifier, we report in Fig. 1 the corrected values of the Fisher information for heterodyne and homodyne detection of quadratures $\hat{x}$ and $\hat{p}$. We show that, as expected, the monitoring of quadrature $\hat{x}$ is still the optimal one; moreover we observe that at longer times, the homodyne monitoring of $\hat{p}$ overcomes the performance of heterodyne detection.

As concerns the estimation of a force acting on a mechanical oscillator, the evolution equation for the derivative of the first moments $\partial_{\theta} \mathbf{R}_{t}$ reported in Appendix C in Eq. (C13) is not correct. Considering the extra term above we obtain

$$
\frac{d\left(\partial_{\lambda} \mathbf{R}_{t}\right)}{d t}=\left[A+\left(\sigma_{t} B+N\right) B^{\top}\right]\left(\partial_{\lambda} \mathbf{R}_{t}\right)+\partial_{\lambda} \mathbf{u}
$$

Because of the extra term, it is not straightforward to obtain the analytical solution of the differential equation, and the formulas obtained in the paper are not correct. However the evolution is still deterministic, and thus, the Fisher information can easily be obtained numerically without needing to average over thousands of trajectories. We observe the same main properties for the Fisher information we discussed in the paper; in particular, we find that the optimal measurement is obtained for $\phi=\pi / 2$, that corresponds to the homodyne monitoring of quadrature $\hat{p}_{c}$. The main qualitative difference is highlighted in Fig. 2 where, as in the paper, we report the behavior of the Fisher information as a function of time and for different values of the loss parameter $\kappa$; we in fact observe that the Fisher information is monotonically increasing with $\kappa$ for all the values of $\omega_{m} t$ we have investigated.

We want to remark that analytical solutions can still be obtained in experimentally relevant scenarios. We report here the example of a single-mode displacement estimation with timecontinuous monitoring described by the following stochastic master equation, that describes continuous monitoring of a levitated nanosphere undergoing momentum diffusion [1,2]:

$$
d \varrho=-i \lambda[\hat{p}, \varrho] d t+\kappa \mathcal{D}[\hat{x}] \varrho d t+\sqrt{\eta \kappa} \mathcal{H}[\hat{x}] \varrho d w
$$

The covariance matrix of the conditional state evolves deterministically as

$$
\sigma_{t}=\left(\begin{array}{cc}
\frac{1}{1+2 \eta \kappa t} & 0 \\
0 & 1+2 \kappa t
\end{array}\right)
$$

In order to evaluate the Fisher information corresponding to the estimation of the parameter $\lambda$, one only needs the vector $\partial_{\lambda}\langle\hat{x}\rangle_{t}$, whose evolution is described by the equation,

$$
\frac{d\left(\partial_{\lambda}\langle\hat{x}\rangle_{t}\right)}{d t}=-\frac{2 \eta \kappa}{1+2 \eta \kappa t}\left(\partial_{\lambda}\langle\hat{x}\rangle_{t}\right)-1
$$

and that can be analytically solved as

$$
\partial_{\lambda}\langle\hat{x}\rangle_{t}=-\frac{1+\eta \kappa t}{1+2 \eta \kappa t} t .
$$

The corresponding infinitesimal and total Fisher information can be evaluated straightforwardly, and one obtains

$$
\begin{aligned}
d F_{t} & =\frac{4 t^{2} \eta \kappa(1+t \eta \kappa)^{2}}{(1+2 t \eta \kappa)^{2}} \\
F_{t} & =\frac{2 t^{3} \eta \kappa(2+t \eta \kappa)}{3(1+2 t \eta \kappa)}
\end{aligned}
$$

We would like also to remark that the formula for the matrix B reported in the paper after Eq. (7) is not correct. It should read $B=C \Omega\left(\sigma_{b}+\sigma_{m}\right)^{-1 / 2}$.

The author would like to thank F. Albarelli for several discussions that contributed to find the error in the paper. 


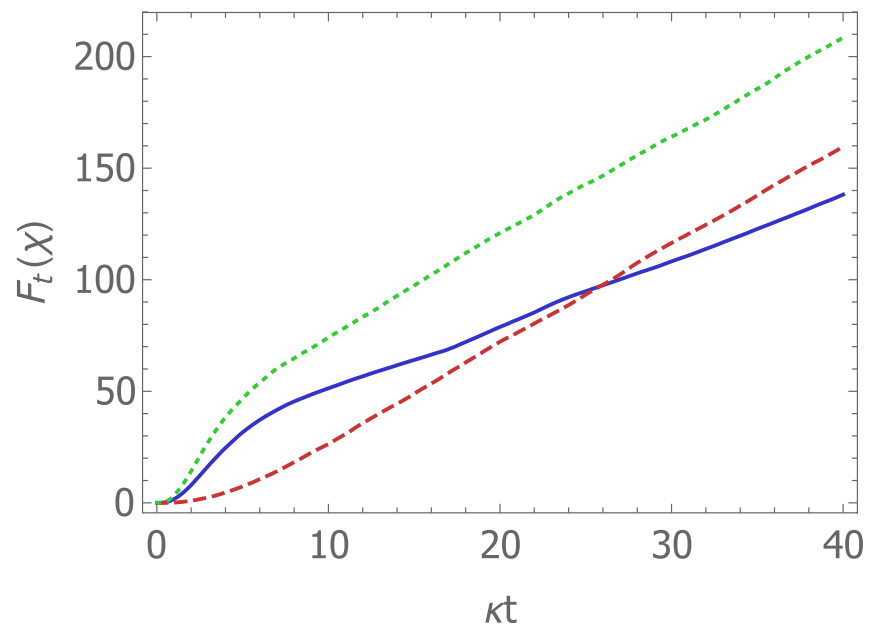

FIG. 1. FI $F_{t}(\chi)$ for continuous homodyne and heterodyne detection as a function of time and for $\chi=-0.2 \kappa$ (numerical evaluation with 2000 trajectories). The green dotted line: homodyne detection of quadrature $\hat{x}$; the red dashed line: homodyne detection of quadrature $\hat{p}$; the blue solid line: heterodyne detection.

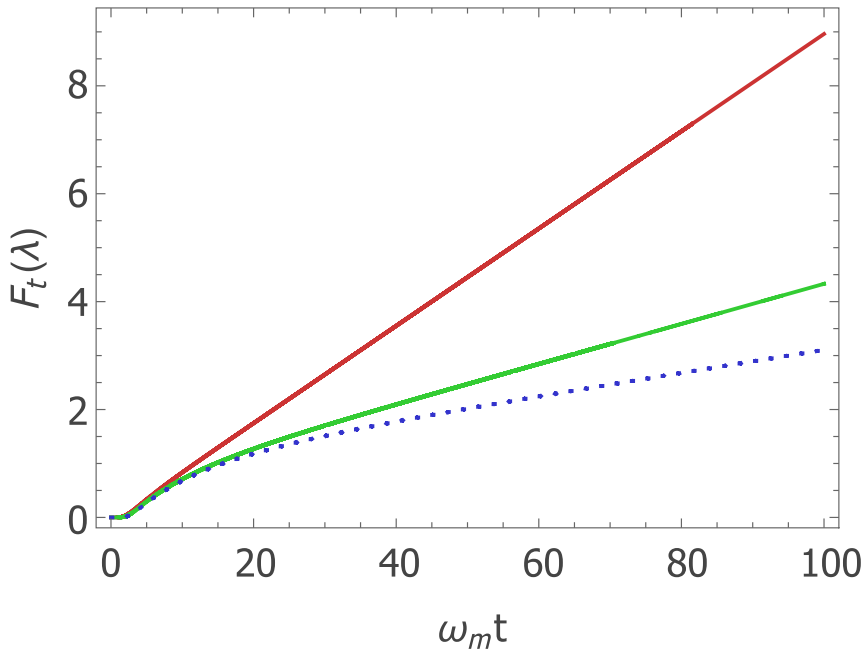

FIG. 2. FI $F_{t}(\lambda)$ for continuous homodyne detection of the cavity field quadrature $\hat{p}_{c}$ as a function of time and for different values of the cavity decay rate: $\kappa=\omega_{m} / 2$ : the red solid line; $\kappa=\omega_{m} / 10$ : the green dashed line; $\kappa=\omega_{m} / 20$ : the blue dotted line (the other parameters are chosen as $g=\omega_{m} / 2, \gamma=\omega_{m} / 3$, and $\eta=1$ ).
[1] A. C. Pflanzer, O. Romero-Isart, and J. I. Cirac, Phys. Rev. A 86, 013802 (2012).
[2] M. G. Genoni, O. S. Duarte, and A. Serafini, New J. Phys. 18, 103040 (2016). 